\title{
Synthesis of New
}

\section{2-Phenylamino-4H-chromene-3-carbonitrile Derivatives and Their Effects on Tumor Cell Lines and against Protein Kinases}

\author{
Ali Bouattour1, Mehdi Fakhfakh1, Souhir Abid1,2, Ludovic Paquinn",4, Rémy Le Guével5, \\ Thierry Charlier5, Sandrine Ruchaud6, Stéphane Bach6 ${ }^{6}$, Jean-Pierre Bazureau ${ }^{3,4 *}$, \\ Houcine Ammar ${ }^{1}$
}

\begin{abstract}
${ }^{1}$ Laboratoire de Chimie Appliquée: Hétérocycles, Corps Gras et Polymères, Faculté des Sciences de Sfax, Université de Sfax, Sfax, Tunisie

${ }^{2}$ Département de Chimie, Collège des Sciences et des Arts, Université de Jouf, Al Qurayyat, KSA

${ }^{3}$ Institut des Sciences Chimiques de Rennes (ISCR), UMR CNRS 6226, Université de Rennes 1, Campus de Beaulieu, Rennes, France

${ }^{4}$ S2 Wave Platform, ScanMAT UMS 2001CNRS, Université de Rennes 1, Campus de Beaulieu, Rennes, France

${ }^{5}$ Université de Rennes 1, ImPACcell Platform, Campus Villejean, Rennes, France

${ }^{6}$ Sorbonne Universités, UPMC Université Paris 06, CNRS USR3151, Protein Phosphorylation and Human Disease Unit, KISSf Platform, Station Biologique, Place Georges Teissier, Roscoff, France

Email: *jean-pierre.bazureau@univ-rennes1.fr
\end{abstract}

How to cite this paper: Bouattour, A., Fakhfakh, M., Abid, S., Paquin, L., Guével, R.L., Charlier, T., Ruchaud, S., Bach, S., Bazureau, J.-P. and Ammar, H. (2020) Synthesis of New 2-Phenylamino- $4 H$-chromene3-carbonitrile Derivatives and Their Effects on Tumor Cell Lines and against Protein Kinases. International Journal of Organic Chemistry, 10, 88-103.

https://doi.org/10.4236/ijoc.2020.102006

Received: February 27, 2020

Accepted: June 27, 2020

Published: June 30, 2020

Copyright () 2020 by author(s) and Scientific Research Publishing Inc. This work is licensed under the Creative Commons Attribution International License (CC BY 4.0).

http://creativecommons.org/licenses/by/4.0/ (c) (i) Open Access

\begin{abstract}
The synthesis of 2-phenylimino- $4 H$-chromene-3-carbonitriles 6(a-d) in good overall yields using an efficient and practical methodology in 3 steps has been implemented in this present work. The first step was a heterocyclization between 2-hydroxybenzaldehyde 1 and propanedinitrile 2 which produced 2 -iminocoumarin 3 which was submitted to nitrogen/nitrogen displacement in the presence of aromatic primary amine 4 . In the third step, reduction of 5 led to the desired 2-phenylimino-4H-chromene-3-carbonitriles 6. Compounds $\mathbf{5}(\mathrm{a}-\mathrm{d})$ and $\mathbf{6}(\mathrm{a}-\mathrm{d})$ were evaluated for their potential in vitro cytotoxicity against six selected tumor cell lines (Huh7-D12, Caco2, MDA-MB231, HCT 116, PC3 and NCI-H727) and tested for their protein kinase inhibition on eight selected protein kinases. Among them, compounds $5 \mathrm{c}$ and $\mathbf{6 b}$ exhibited inhibition on $\mathrm{HsCK}_{\mathrm{Se}}(5 \mathrm{c}$ : $44 \%$ and $6 \mathrm{~b}: 42 \%$ at $1 \mu \mathrm{M}$ ) and $5 \mathrm{c}$ for cytotoxicity on PC3 cell lines (63\% at $25 \mu \mathrm{M})$.
\end{abstract}

\section{Keywords}

Iminocoumarin, $2 H$-[1]benzopyran, 2-Imino- $2 H$-[1]benzopyran, 
4H-chromene, 2-Amino- $4 H$-chromene, Nitrogen/Nitrogen Displacement, Protein Kinase Inhibition, Cytotoxicity

\section{Introduction}

During the two last decades, the 2-amino- $4 H$-chromene moieties (Figure 1) emerged to be a promising platform in new chromene and benzo chromene derivatives, which exhibit a wide range of biological and pharmacological activities. For examples, Crolibulin ${ }^{\mathrm{TM}}$ (EPC 2407) (I) was currently in phase I/II of clinical trials in 2016 for the treatment of aggressive and advanced solid tumors [1] [2] [3] [4]. During the randomized phase II trial (NTC01240590) [5], the authors compared the activity of the combination of Crolibulin ${ }^{\mathrm{TM}}$ plus cisplatin with cisplatin alone on a maximum of 40 enrolled patients with a focus on anaplastic thyroid cancer (ATC). MX 58151 (II) or 2-amino-4-(3-bromo-4,5-dimethoxyphenyl)-4Hchromene-3-carbonitrile was identified as a tubulin inhibitor [6]. It induced apoptosis with an $\mathrm{EC}_{50}$ of $50 \mathrm{nM}$ and inhibited cell growth with a $\mathrm{GI}_{50}$ of $37 \mathrm{nM}$ in T47D breast cancer cells [7]. HA 14-1 (III) is another small molecule and nonpeptidic ligand of Bcl-2 surface pocket discovered by using the de novo computer-aided design strategy based on the predicted structure of Bcl-2 protein [8]. In vitro binding studies of HA 14-1 (III) against a set of Jurkat cells confirmed inhibition of Bcl-2 protein [9] [10] and the authors of this study demonstrated that the 6-bromo on (III) is not essential for its bioactivity and the 6-position can accommodate a variety of alkyl and aryl functional groups [11]. SV30 or ethyl [2-amino-6-bromo-4-diethylmalonate]-4H-chromene-3-carboxylate (IV), an analogue of pro-apoptotic molecule HA 14-1 (III), was explored in combination with lipid nanocapsules (LNCs) on F98 cells to improve its biological activities [12]. The authors demonstrated that SV30-LNCs were able to trigger cell death together with a potentiation of the mitochondrial membrane potential decrease. The naphthopyran LY 290181 (V) [2-amino-4-(pyridyl)-4Hnaphtho[1,2-b]pyran-3-carbonitrile] was identified in 1997 as a potent antiproliferative compound blocking cells in the $\mathrm{G}_{2} / \mathrm{M}$ phase of the cell cycle associated to action on microtubules [13] [14]. Finally, it's interesting to note that a QSAR (Qualitative Structure Activity Relationship) analysis was developed with novel 3D-descriptors using Triplets Of Pharmacophoric Points (TOPP) on 80 virtual apoptosis inducing 3-amino-4-aryl-4H-chromene-3-carbonitriles [15]; the underlying idea of this work was to optimize the pharmacological and pharmacokinetic properties via QSAR models prior to synthesis.

As part of our program aimed at developing new methods, new building-blocks and platforms for the preparation of heterocyclic compounds showing potential biological properties for central nervous system (CNS) (Alzheimer's disease and Down syndrome) [16] [17], or for cancer (calcium ion channel inhibitors in cancer) [18], we were motivated in this work by the synthetic development of some new 2-amino-4H-[1]-chromene-3-carbonitriles without aryl 


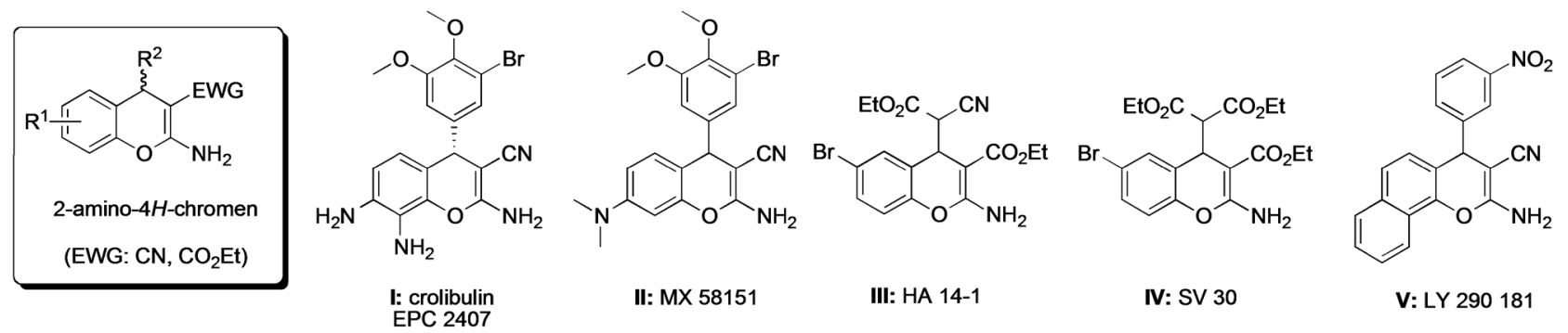

Figure 1. Structure of bioactive 2 -amino- $4 H$-chromene derivatives.

substituent in C-4 position but with a phenyl fragment on the 2-amino function, to evaluate their biological activities on protein kinases (PKs) and also their anti-proliferative activities on tumor cell lines.

\section{Results and Discussion}

\subsection{Chemistry}

For this project, the desired 2-N-phenylamino-4H-chromene-3-carbonitriles 6(a-d) were prepared only in three steps (Scheme 1). The first step involved synthesis of iminocoumarins $3(\mathrm{a}, \mathrm{b})$ or 2-imino-2H-[1]-benzopyran-3-carbonitriles and protocol of this classical heterocyclization was developed in our laboratory [19] [20] [21]. The reaction was realized from an equimolecular mixture of 2-hydroxybenzaldehyde 1 (1a: 2-hydroxy-3-methoxybenzaldehyde or $o$-vanillin, 1b: 2-hydroxynaphthaldehyde) and propanedinitrile 2 using $0.5 \%$ of piperidine in ethanol at room temperature. After a reaction time of 9 hours, the volatile compounds were eliminated in vacuo and we obtained these starting iminocoumarins in good yields (3a: 85\% [19], 3b: 90\% [22]). For the second step, the transformation of the 2-imino-2H-[1]-benzopyran-3-carbonitriles $3(\mathrm{a}, \mathrm{b})$ into 2- $N$-phenylimino-2 $H$-[1]-benzopyran-3-carbonitriles $\mathbf{5}(\mathrm{a}-\mathrm{d})$ is the key step in our approach for introduction of molecular diversity in this C-2 position. In a preliminary approach, we studied this nitrogen/nitrogen displacement with volatile primary aliphatic amine 4 (from 1 to 2 equivalents) using solvent-less reaction conditions [23] in a mono-mode microwave cavity (Monowave ${ }^{\circledR} 300$ Anton-Paar apparatus operating at a frequency of $2.45 \mathrm{GHz}$ from 0 to $800 \mathrm{Watt}$ ). Initial attempts to obtain a good reproducibility for this nitrogen/nitrogen displacement with primary aliphatic amine 4 involving modification of the reaction conditions (reaction time range: 15 - 60 min., ratio of reagents 3/4: from 1 to 2, reaction temperature, use of non-polar or polar solvents) were unsuccessful. Therefore, we decided to abandon this solvent-less microwave approach.

On the other hand, the use of an aromatic primary amine $4(a, b)$ associated with an acidic catalysis was much fruitful. Indeed, when this reaction was carried out in glacial acetic acid with an equimolecular mixture of $\mathbf{3}$ and $\mathbf{4}$ at room temperature, we observed that after 4 hours, the desired compound 5 obtained by nitrogen/nitrogen displacement (Scheme 2) becomes insoluble in the reaction medium. Thus recovery of this insoluble material by filtration on a Buchner 


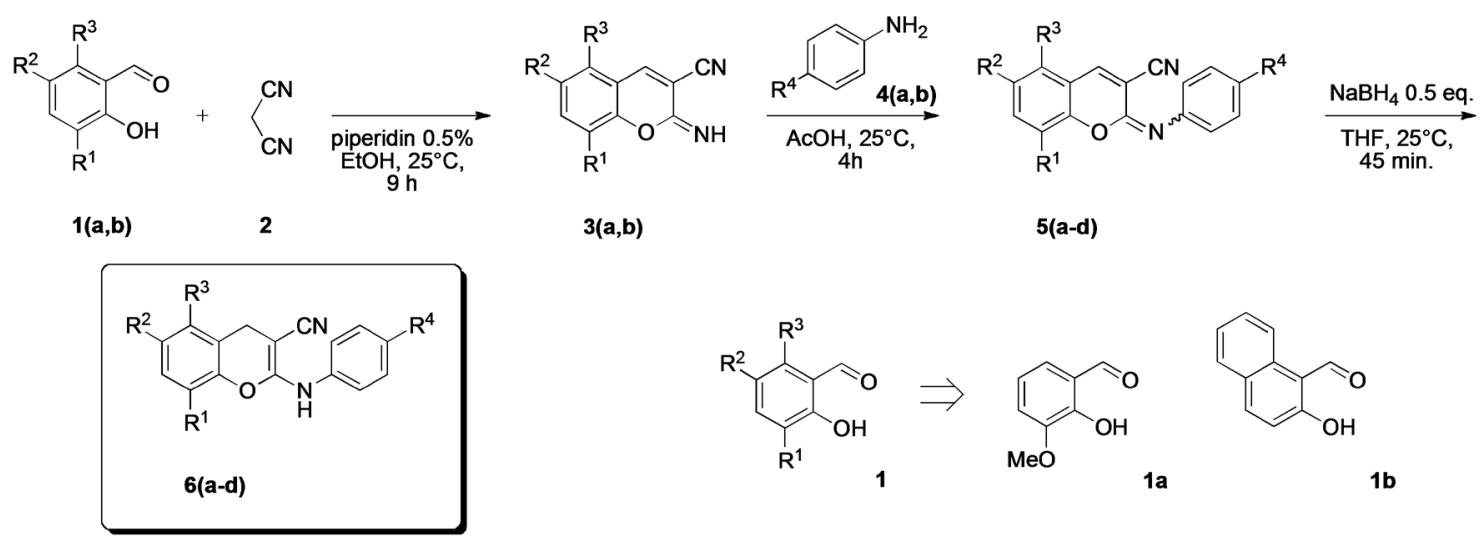

Scheme 1. Route used for the synthesis of 2- $N$-phenylamino- $4 H$-chromene-3-carbonitrile derivatives 6(a-d).

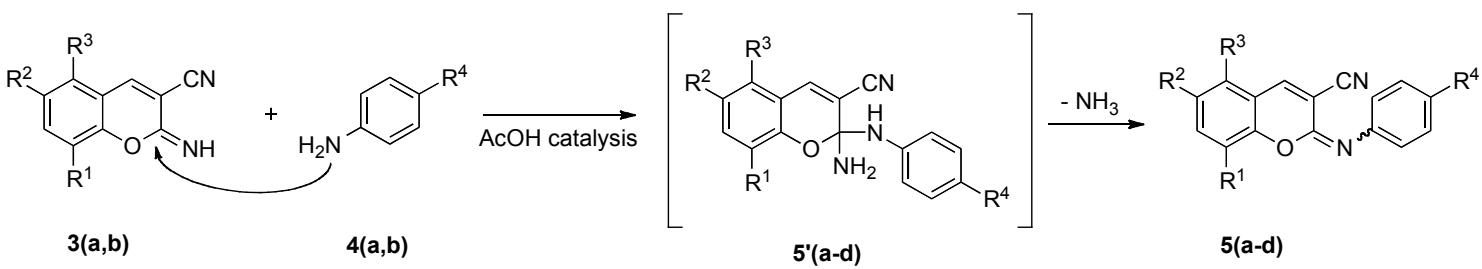

Scheme 2. A plausible suggested mechanism for nitrogen/nitrogen displacement via a nucleophilic attack of amino group of 4 to C-2 imino function of 3 .

funnel followed by washing with deionized water allows us to get very easily the four desired 2- $N$-phenylimino-2 $H$-[1]-benzopyran-3-carbonitriles $5(\mathrm{a}-\mathrm{d})$ in yields ranging from $55 \%$ to $80 \%$ (Table 1 ). ${ }^{1} \mathrm{H}$ NMR control in DMSO- $d_{6}$ of 5 (a-d) after filtration showed that no need of supplementary purification and it can be used as it in the next step. Finally for the third step involving transformation of 2- $\mathrm{N}$-phenylimino-2 $\mathrm{H}$-[1]-benzopyran-3-carbonitriles 5(a-d) into 2- $\mathrm{N}$-phenylamino- $4 H$-chromene-3-carbonitriles 6(a-d), we used an experimental protocol developed in our laboratory [19] but only changing the nature of the solvent reaction (THF vs $\mathrm{MeOH}$ ) and the reaction temperature. From 0.5 equivalent of sodium borohydride in THF at $25^{\circ} \mathrm{C}$ during $45 \mathrm{~min}$., the desired compounds 6(a-d) were obtained in good yields (Table 1) after purification ( $81 \%-89 \%)$.

Before exploring their potential biological activities, the four products $5(\mathrm{a}-\mathrm{d})$ and their four reduced derivatives $6(\mathrm{a}-\mathrm{d})$ were characterized by ${ }^{1} \mathrm{H},{ }^{13} \mathrm{C} \mathrm{NMR}$, HRMS and FTIR. In the IR spectrum, the presence of carbonitrile function in C-3 position was detected at $2227-2229 \mathrm{~cm}^{-1}$ for 5 (a-d) and at $2189-2195 \mathrm{~cm}^{-1}$ for 6(a-d) (Table 1). The C-4 imino functions of 5(a-d) were analyzed at 1650 $1662 \mathrm{~cm}^{-1}$. Important absorption bands were observed at $3278-3320 \mathrm{~cm}^{-1}$ for the $\mathrm{NH}$ stretching frequencies of $6(\mathrm{a}-\mathrm{d})$. In ${ }^{1} \mathrm{H}$ NMR spectrum of compounds 6(a-d), a signal located at $3.54<\delta<3.98 \mathrm{ppm}$ is in agreement with the H-4 methylene fragment. In ${ }^{13} \mathrm{C}$ NMR spectrum, the signal for the $\mathrm{CH}_{2}$ fragment in position C-4 of compounds $6(\mathrm{a}-\mathrm{d})$ is located at $22.4<\mathrm{d}<25.3 \mathrm{ppm}$. For HRMS analysis, the $[\mathrm{M}+\mathrm{Na}]^{+}$molecular ion signal for all products 5 and $\mathbf{6}$ were obtained as base signal. 
Table 1. Results obtained for the preparation of 2- $N$-phenylimino- $2 H$-[1]-benzopyran-3carbonitrile 5(a-d) and 2-N-phenylamino-4H-chromene-3-carbonitrile 6(a-d).

\begin{tabular}{|c|c|c|c|c|c|c|}
\hline & \multirow{2}{*}{ Compound } & \multirow{2}{*}{ Yield $(\%)^{\mathrm{a}}$} & \multirow{2}{*}{$\mathrm{mp}\left({ }^{\circ} \mathrm{C}\right)$} & \multicolumn{3}{|c|}{ IR $\left(\mathrm{en} \cdot \mathrm{cm}^{-1}\right)$} \\
\hline & & & & $\mathrm{n}_{(\mathrm{C}=\mathrm{N})}$ & $\mathrm{n}_{\text {(nitrile) }}$ & $\mathrm{n}_{(\mathrm{NH})}$ \\
\hline $5 a$ & & 60 & $174-176$ & 1650 & 2227 & - \\
\hline $5 b$ & & 70 & $180-182$ & 1650 & 2227 & - \\
\hline $5 c$ & & 55 & $196-198$ & 1662 & 2229 & - \\
\hline $5 \mathrm{~d}$ & & 80 & $232-234$ & 1650 & 2229 & - \\
\hline $6 a$ & & 81 & $122-124$ & - & 2195 & 3320 \\
\hline $6 \mathrm{~b}$ & & 89 & $148-150$ & - & 2191 & 3232 \\
\hline $6 c$ & & 84 & $188-190$ & - & 2193 & 3278 \\
\hline $6 d$ & & 85 & $200-202$ & - & 2189 & 3235 \\
\hline
\end{tabular}

${ }^{a}$ Yield of isolated product.

\subsection{Biology}

For the second part of this project related to $2-N$-phenylamino- $4 H$-chromene-3carbonitrile $\mathbf{6}(\mathrm{a}-\mathrm{d})$ and their precursors $5(\mathrm{a}-\mathrm{d})$, we have been interested in exploring their potential biological properties, particularly their impact on protein kinase inhibition activity and their cytotoxic character against various tumor cell lines.

The new compounds $\mathbf{5}$ and $\mathbf{6}$ were evaluated on eight different in vitro kinase assays. The selected enzymes are very important in protein phosphorylation of serine, threonine and tyrosine residues, which are connected, in many cellular regulatory mechanisms; dysfunction of protein kinase activities is in many cases responsible for human diseases. The protein kinases used for these in vitro assays are respectively HsCDK5-p25 (Homo sapiens cyclin-dependent kinase 5p-25) [24], HsCDK9/cyclin T (Homo sapiens cyclin T dependent kinase 9 pro- 
tein) [25], $S s c C K 1 \varepsilon$ (casein kinase $1 \varepsilon$, from porcine brain) [26], $S s c G S K 3 \beta$ (glycogen synthase kinase-3 $\beta$, from porcine brain) [27], HsPim1 (Homo sapiens Pim1 proto-oncogene, serine/threonine kinase) [28], MmCLK1 (from Mus musculus, Dual specificity protein kinase) [29] and RnDYRK1A-kd (Rattus norvegicus, Dual specificity tyrosine phosphorylation regulated kinase 1A) [30].

Results for these in vivo kinase assays are reported in Table 2 with the rate (\%) of residual activities at $10 \mathrm{mM}$ and $1 \mathrm{mM}$. These results showed three categories of compounds. The first concerns compounds with no significant protein kinase activity (rate $>60 \%-70 \%$ at $10 \mu \mathrm{M}$ and $1 \mu \mathrm{M}$ ). The second category integrated compounds with moderate interest because the rate is less than or close to $50 \%$ and for the third category; the rate is less than $50 \%$ at $10 \mu \mathrm{M}$ and $1 \mu \mathrm{M}$. In this last one, the tested compounds 5 or $\mathbf{6}$ have a marked effect on the catalytic activity. Examination of results given in Table 2 showed that many compounds (Figure 2) exhibited inhibitory activity on $H_{s} \mathrm{CK} 1 \mathrm{e}, \mathrm{HsHaspin}, \mathrm{HsCDK} 9 / \mathrm{cyclin}$ T. This concerned respectively and particularly $H s \mathrm{CK} 1 \mathrm{e}$ with $5 \mathrm{c}(41 \%$ at $1 \mu \mathrm{M})$, 5d $(57 \%$ at $1 \mu \mathrm{M})$ and $6 \mathrm{c}(46 \%$ at $10 \mu \mathrm{M}$ and $59 \%$ at $1 \mu \mathrm{M})$. For $H s$ Haspin, the

Table 2. Effects of 2-phenylimino-2 $H$-benzopyran-3-carbonitrile 5(a-d) and 2- $N$-phenylamino- $4 H$-chromene-3-carbonitrile $6(\mathrm{a}-\mathrm{d})$ on the catalytic activity of eight protein kinases.

\begin{tabular}{|c|c|c|c|c|c|c|c|c|c|}
\hline \multicolumn{10}{|c|}{ Primary screening (\% of residual activity at $10 \mathrm{mM}$ and $1 \mathrm{mM}$ ) } \\
\hline Compound & $\begin{array}{l}\text { Conc. } \\
(\mathrm{mM})\end{array}$ & $\begin{array}{l}\text { CDK5/ } \\
\text { p25 }\end{array}$ & $\begin{array}{l}\text { CDK9/ } \\
\text { cyclin T }\end{array}$ & CK1e & GSK3b & Haspin & PIM1 & CLK1 & DYRK1A \\
\hline \multirow[b]{2}{*}{$5 a$} & 10 & $\geq 100$ & 96 & 60 & 79 & 55 & 96 & 80 & 71 \\
\hline & 1 & 98 & 76 & 65 & 82 & 91 & 84 & 88 & 99 \\
\hline \multirow{2}{*}{$5 b$} & 10 & 98 & 84 & 78 & 85 & 68 & 97 & 78 & $\geq 100$ \\
\hline & 1 & 95 & 61 & 52 & 90 & $\geq 100$ & 99 & 87 & 85 \\
\hline \multirow{2}{*}{$5 c$} & 10 & 98 & 72 & 63 & 78 & 60 & 72 & 90 & 90 \\
\hline & 1 & 94 & 57 & 44 & 72 & 45 & 81 & 80 & 58 \\
\hline \multirow{2}{*}{$5 d$} & 10 & 91 & 51 & 38 & 65 & 34 & 79 & 81 & 82 \\
\hline & 1 & 94 & 54 & 57 & 82 & 51 & 78 & 75 & 57 \\
\hline \multirow{2}{*}{$6 a$} & 10 & 86 & 62 & 58 & 66 & 46 & 52 & 51 & 34 \\
\hline & 1 & $\geq 100$ & 60 & 63 & 74 & 67 & 84 & 83 & 71 \\
\hline \multirow{2}{*}{$6 \mathrm{~b}$} & 10 & 76 & 57 & 47 & 71 & 53 & 68 & 74 & 65 \\
\hline & 1 & 86 & 50 & 42 & 65 & 90 & 92 & 75 & 67 \\
\hline \multirow{2}{*}{$6 c$} & 10 & 80 & 43 & 46 & 55 & 38 & 72 & 81 & 74 \\
\hline & 1 & 89 & 54 & 59 & 64 & 71 & 80 & 71 & 78 \\
\hline \multirow{2}{*}{$6 d$} & 10 & 87 & 38 & 39 & 31 & 31 & 76 & 61 & $\geq 100$ \\
\hline & 1 & 89 & 63 & 67 & 78 & 66 & 77 & 71 & 88 \\
\hline
\end{tabular}

NB: $\geq 100$, indicates that the compound cannot inhibit the enzymatic activity at the tested concentration. $\square$ : The compound has an interest on the catalytic activity because the rate is less than or close to $50 \%$ : The compound has a marked effect on the catalytic activity because the rate is less than $50 \%$. 
<smiles>Cc1cc2c(ccc3ccccc32)o/c1=N\c1ccccc1</smiles>

Hs CK1 1 : $44 \%(1 \mu \mathrm{M})$ Hs Haspin: $45 \%(1 \mu \mathrm{M})$

HCT 116: $20 \%$; PC3: $63 \%$ $\mathrm{NCl}-\mathrm{H} 727: 41 \%$ Caco $2=55 \%$<smiles>COc1ccc(/N=c2\oc3ccc4ccccc4c3cc2C)cc1</smiles>

$5 d$

Hs CK1 1 : $38 \%(10 \mu \mathrm{M}): 57 \%(1 \mu \mathrm{M})$ Hs Haspin: $34 \%(10 \mu \mathrm{M}) ; 51 \%(1 \mu \mathrm{M})$

Caco 2: $44 \%$

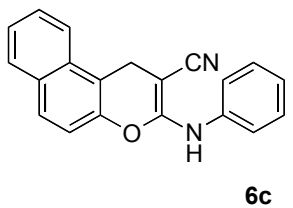

Hs CK1ع: 46\% (10 $\mu \mathrm{M}) ; 59 \%(1 \mu \mathrm{M})$ Hs CDK9/cyclinT: $43 \%$ (10 $\mu \mathrm{M}) ; 54 \%(1 \mu \mathrm{M})$

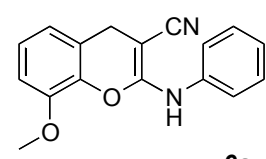

Hs Haspin: 46\% (10 $\mu \mathrm{M})$ Rn DYRK1A: 34\% (10 $\mu \mathrm{M})$<smiles>COc1ccc(NC2=C(C#N)Cc3cccc(OC)c3O2)cc1</smiles>

6b

Hs CK1ع: 47\% (10 $\mu \mathrm{M}) ; 42 \%(1 \mu \mathrm{M})$ Hs Haspin: $53 \%(10 \mu \mathrm{M})$

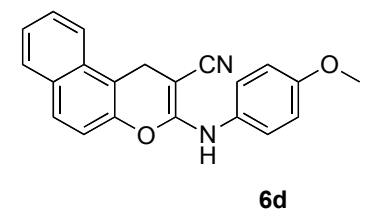

Hs CDK9/cyclinT: $38 \%(10 \mu \mathrm{M})$

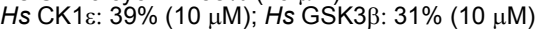
Hs Haspin: $31 \%(10 \mu \mathrm{M})$

PC3: $61 \%$

Figure 2. 2-Phenylimino-2 $H$-[1]-benzopyran-3-carbonitrile 5(c, d) and 2-phenylamino-4H-chromene-3-carbonitrile 6(a-d) which are bioactive on tumor cell lines or against protein kinases.

better result was obtained with $5 \mathrm{c}$ : $45 \%$ at $1 \mu \mathrm{M}$. For $R n \mathrm{DYRK} 1 \mathrm{~A}$ and $H s \mathrm{GSK} 3 \mathrm{~b}$, good residual activities were observed only at $10 \mu \mathrm{M}$ for $6 \mathrm{a}$ (RnDYK1A: $34 \%$ ) and 6d (HsGSK3b: 31\%).

Pursuing this biological exploration, the 2-phenylimino- $H$-[1]-benzopyran3-carbonitrile 5(a-d) and 2-phenylamino- $4 H$-chromene-3-carbonitrile 6(a-d) were also subjected for their in vitro cytotoxic potential on six selected human cancer cells which are respectively: Huh7 for hepatocellular carcinoma, Caco2 for colorectal adenocarcinoma, MDA-MB231 for human breast adenocarcinoma, HCT 116 for colon carcinoma, PC3 for human Caucasian prostate adenocarcinoma and NCI-H727 for human lung non-small cell carcinoma. For each tumoral cell line, the $\%$ of cell survival was measured at a single dose of $25 \mu \mathrm{M}$ (after $48 \mathrm{~h}$ ) in triplicate. Roscovitine, doxorubicin and taxol were used as references for positive control. It can be observed from these primary screening results reported in Table 3 that only two compounds exhibited antiproliferative activities in tumoral cell lines. This concerns $\mathbf{5 c}$, which exhibited cytotoxic effect on HCT 116 (20\% of survival), PC3 (63\%) and NCI-H727 (41\%). The presence of a methoxy group on the 2-phenylimino moiety in $\mathbf{5 d}$ increase the percentage of survival, excepted on Caco2 (44\%). For compound $6 \mathrm{~d}$, which is the reduced derivative of $\mathbf{5 d}$, it showed cytotoxicity against PC3 (61\%).

\section{Conclusion}

This preliminary study described a practical approach to $2-\mathrm{N}$-phenylamino- $4 \mathrm{H}$ chromene-3-carbonitriles 6(a-d) in three steps. The key step in this methodology is the nitrogen/nitrogen displacement between 2-imino- $2 H$-[1]-benzopyran3 -carbonitriles $5(\mathrm{a}-\mathrm{d})$ and aromatic primary amines $4(\mathrm{a}, \mathrm{b})$ in acetic medium on the 2 -imino function. Insolubility of compounds $5(\mathrm{a}-\mathrm{d})$ in the acetic acid mixture 
Table 3. Antiproliferative activity of 2-phenylimino- $2 H$-benzopyran-3-carbonitrile 5(a-d) and 2-phenylamino- $4 H$-chromene-3-carbonitrile $6(\mathrm{a}-\mathrm{d})$ on six representative tumor cell lines.

\begin{tabular}{|c|c|c|c|c|c|c|}
\hline \multirow{2}{*}{ Compound } & \multicolumn{6}{|c|}{$\%$ of survival measured at $25 \mathrm{mM}$ (after $48 \mathrm{~h}$ using a single dose, triplicate) } \\
\hline & Huh7 D12 & Caco 2 & MDA-MB231 & HCT 116 & PC3 & NCI-H727 \\
\hline $5 a$ & 98 & 100 & 111 & 115 & 88 & 109 \\
\hline $5 b$ & 95 & 89 & 110 & 64 & 83 & 79 \\
\hline $5 c$ & 71 & 55 & 78 & 20 & 63 & 41 \\
\hline $5 \mathrm{~d}$ & 61 & 44 & 65 & 54 & 60 & 54 \\
\hline $6 a$ & 103 & 92 & 103 & 97 & 95 & 91 \\
\hline $6 \mathrm{~b}$ & 83 & 98 & 118 & 119 & 87 & 101 \\
\hline $6 c$ & 79 & 87 & 90 & 99 & 85 & 98 \\
\hline $6 d$ & 86 & 90 & 82 & 74 & 61 & 91 \\
\hline DMSO & 100 & 100 & 100 & 100 & 100 & 100 \\
\hline Roscovitine & 45 & 21 & 38 & 7 & 19 & 9 \\
\hline Doxorubicin & 64 & 79 & 46 & 23 & 37 & 31 \\
\hline Taxol & 50 & 78 & 50 & 9 & 26 & 26 \\
\hline
\end{tabular}

NB: $\square$ : The compound has antiproliferative activity on tumor cell but the survival rate is less than or close to 50\%; $\square$ : The compound has a marked antiproliferative activity on tumor cell and the survival rate is less than $50 \%$.

facilitated its separation by simple filtration, which offers a practical, attractive protocol and extension to a wide variety of aromatic primary amine 4 is possible. The explorations of biological activities were successively performed on a panel of six tumoral cell lines and against eight protein kinases. Among the bioactive compounds, $5 \mathrm{c}$ and $\mathbf{6 b}$ turned out to be interesting because they present a good percentage of residual activities at $1 \mu \mathrm{M}$ for $H_{s} \mathrm{CK} 1 \mathrm{e} . \mathbf{5 c}$ showed also a marked antiproliferative activity on HCT 116 (20\% of survival). The present work is the starting point of a new longer program through a next structure activity relationship (RSA) study for a complete identification of a better promising anticancer agent bearing a 2-amino- $4 \mathrm{H}$-chromene platform.

\section{Experimental Section}

\subsection{Chemistry}

General information: Solvents were evaporated with a BUCHI rotary evaporator. All reagents and solvents were purchased from Acros Fisher, Sigma-Aldrich Chimie, and Fluka France and were used without further purification. ${ }^{1} \mathrm{H}$ NMR spectra were recorded on BRUKER Avance $300(300 \mathrm{MHz})$ spectrometer and ${ }^{13} \mathrm{C}$ NMR spectra on BRUKER Avance $300(75 \mathrm{MHz})$ spectrometer. The high resolution mass spectra (HRMS) were recorded in positive mode using direct Electrospray infusion, respectively on a Waters Q-Tof 2 or on a Thermo Fisher Scientific Q-Exactive spectrometers at the "Centre Régional de Mesures Physiques de 
l'Ouest" platform and centesimal analysis of the final compounds was performed on a microanalyzer Thermo Scientific Flash EA1112 CHNS/O at the CRMPO platform, ScanMAT UMS CNRS 2001, Rennes, France). IR spectra were registered on a Jasco FT-IR 420 spectrophotometer using $\mathrm{KBr}$ pellets. Melting points were determined on a Kofler melting point apparatus and were uncorrected. 2-Imino-2 $H$-[1]-benzopyran-3-carbonitrile 3(a, b) were synthesized according to procedure described in literature [20] [22].

Standard procedure for the synthesis of 2- $\mathrm{N}$-phenylimino-2H-[1]-benzopyran3-carbonitrile 5(a-d).

To a stirred solution of 2-imino-2H-1-benzopyrane-3-carbonitriles 3 (1 $\mathrm{mmol})$ in glacial acetic acid $(2 \mathrm{ml})$ was added the primary amine $4(1 \mathrm{mmol})$. The resulting mixture was vigorously stirred $(500 \mathrm{rpm})$ at room temperature during 4 hours. The desired product 5 was collected by filtration on a Buchner funnel (porosity No. 4) and washed with deionized water $(3 \times 10 \mathrm{ml})$. The desired 2-phenylimino-2 $H$-1-benzopyrane-3-carbonitriles 5 was dried under high vacuum $\left(10^{-2}\right.$ torr $)$ at $25^{\circ} \mathrm{C}$ for $1 \mathrm{~h}$ to give a powder and then used without purification.

\section{8-Methoxy-2-N-phenylimino-2H-[1]-benzopyran-3-carbonitrile (5a)}

Yield $=60 \%$. Yellowish powder, $\mathrm{mp}=174^{\circ} \mathrm{C}-176^{\circ} \mathrm{C}$. IR $\left(\mathrm{cm}^{-1}\right): v=1650$ $(\mathrm{C}=\mathrm{N}), 2227(\mathrm{C} \equiv \mathrm{N}) ;{ }^{1} \mathrm{H}$ NMR $\left(300 \mathrm{MHz}, \mathrm{DMSO}-d_{6}\right) \delta: 3.81\left(\mathrm{~s}, 3 \mathrm{H}, \mathrm{OCH}_{3}\right), 7.22$ (m, $\left.4 \mathrm{H}, \mathrm{H}_{5}, \mathrm{H}_{6}, \mathrm{H}_{7}, \mathrm{H}_{4^{\prime}}\right), 7.38\left(\mathrm{~d},{ }^{3} \mathrm{~J}=3 \mathrm{~Hz}, 4 \mathrm{H}, \mathrm{H}_{2^{\prime}}, \mathrm{H}_{3^{\prime}}\right), 8.40\left(\mathrm{~s}, 1 \mathrm{H}, \mathrm{H}_{4}\right) ;{ }^{13} \mathrm{C}$ NMR (75 MHz, DMSO- $\left.d_{6}\right) \delta: 56.5\left(\mathrm{OCH}_{3}\right), 105.7\left(\mathrm{C}_{3}\right), 115.0(\mathrm{C} \equiv \mathrm{N}), 117.2\left(\mathrm{C}_{7}\right)$, $118.2\left(\mathrm{C}_{10}\right), 120.5\left(\mathrm{C}_{5}\right), 123.7\left(\mathrm{C}_{2^{\prime}}\right), 124.7\left(\mathrm{C}_{4^{\prime}}\right), 124.9\left(\mathrm{C}_{6}\right), 128.6\left(\mathrm{C}_{3^{\prime}}\right), 142.3\left(\mathrm{C}_{9}\right)$, $144.1\left(\mathrm{C}_{1}\right), 144.7\left(\mathrm{C}_{8}\right), 146.4\left(\mathrm{C}_{2}\right), 146.5\left(\mathrm{C}_{4}\right)$. HRMS, $\mathrm{m} / \mathrm{z} .299 .0792$ found (calculated for $\mathrm{C}_{17} \mathrm{H}_{12} \mathrm{~N}_{2} \mathrm{O}_{2} \mathrm{Na}[\mathrm{M}+\mathrm{Na}]^{+}$requires 299.0792). Anal. Calcd for $\mathrm{C}_{17} \mathrm{H}_{12} \mathrm{~N}_{2} \mathrm{O}_{2}$ : C, 73.90; H, 4.38; N, 10.14. Found C, 73.92; H, 4.40; N, 10.15 .

8-Methoxy-2 -(4-methoxy-phenylimino) -2H-[1]-benzopyran-3-carbonitri $\operatorname{le}(5 b)$

Yield $=70 \%$. Reddish powder, $\mathrm{mp}=180^{\circ} \mathrm{C}-182^{\circ} \mathrm{C}$. IR $\left(\mathrm{cm}^{-1}\right): v=1650$ $(\mathrm{C}=\mathrm{N}), 2227(\mathrm{C} \equiv \mathrm{N}) ;{ }^{1} \mathrm{H}$ NMR $\left(300 \mathrm{MHz}, \mathrm{DMSO}-d_{6}\right) \delta: 3.79\left(\mathrm{~s}, 3 \mathrm{H}, \mathrm{H}_{4^{\prime \prime}}\right), 3.89(\mathrm{~s}$, $\left.3 \mathrm{H}, \mathrm{OCH}_{3}\right), 6.95\left(\mathrm{~d},{ }^{3} J=9 \mathrm{~Hz}, 2 \mathrm{H}, \mathrm{H}_{3}\right), 7.15\left(\mathrm{~d},{ }^{3} J=9 \mathrm{~Hz}, 1 \mathrm{H}, \mathrm{H}_{7}\right), 7.23\left(\mathrm{t},{ }^{3} J=9\right.$ $\left.\mathrm{Hz}, 1 \mathrm{H}, \mathrm{H}_{6}\right), 7.31\left(\mathrm{~d},{ }^{3} J=9 \mathrm{~Hz}, 1 \mathrm{H}, \mathrm{H}_{5}\right), 7.51\left(\mathrm{~d},{ }^{3} J=9 \mathrm{~Hz}, 2 \mathrm{H}, \mathrm{H}_{2}\right), 8.32(\mathrm{~s}, 1 \mathrm{H}$, $\left.\mathrm{H}_{4}\right) ;{ }^{13} \mathrm{C}$ NMR $\left(75 \mathrm{MHz}, \mathrm{DMSO}-d_{6}\right) \delta: 55.2\left(\mathrm{C}_{4^{\prime \prime}}\right), 56.5\left(\mathrm{OCH}_{3}\right), 106.2\left(\mathrm{C}_{3}\right), 113.8$ $\left(\mathrm{C}_{3^{3}}\right), 115.1(\mathrm{C} \equiv \mathrm{N}), 116.8\left(\mathrm{C}_{7}\right), 118.2\left(\mathrm{C}_{10}\right), 120.3\left(\mathrm{C}_{5}\right), 124.9\left(\mathrm{C}_{6}\right), 126.1\left(\mathrm{C}_{2^{2}}\right)$, $136.7\left(\mathrm{C}_{1^{\prime}}\right), 142.3\left(\mathrm{C}_{9}\right), 143.4\left(\mathrm{C}_{8}\right), 145.4\left(\mathrm{C}_{2}\right), 146.4\left(\mathrm{C}_{4}\right), 156.9\left(\mathrm{C}_{4}\right)$. HRMS, $m / z$. 329.0899 found (calculated for $\mathrm{C}_{18} \mathrm{H}_{14} \mathrm{~N}_{2} \mathrm{O}_{3} \mathrm{Na}[\mathrm{M}+\mathrm{Na}]^{+}$requires 329.0897). Anal. Calcd for $\mathrm{C}_{18} \mathrm{H}_{14} \mathrm{~N}_{2} \mathrm{O}_{3}$ : C, 70.58; H, 4.61; N, 9.15. Found C, 70.60; H, 4.62; $\mathrm{N}, 9.15$.

\section{2-N-Phenylimino-3 H-naphtho[2,1-b] pyran-2-carbonitrile (5c)}

Yield $=55 \%$. Yellowish powder, $\mathrm{mp}=196^{\circ} \mathrm{C}-198^{\circ} \mathrm{C} . \mathrm{IR}\left(\mathrm{cm}^{-1}\right): v=1662$ $(\mathrm{C}=\mathrm{N}), 2229(\mathrm{C} \equiv \mathrm{N}) ;{ }^{1} \mathrm{H}$ NMR $\left(300 \mathrm{MHz}, \mathrm{CDCl}_{3}\right) \delta: 7.20\left(\mathrm{t},{ }^{3} J=6 \mathrm{~Hz}, 1 \mathrm{H}, \mathrm{H}_{4}\right)$, $7.23\left(\mathrm{~d},{ }^{3} J=6 \mathrm{~Hz}, 1 \mathrm{H}, \mathrm{H}_{8}\right), 7.34\left(\mathrm{~d},{ }^{3} J=6 \mathrm{~Hz}, 2 \mathrm{H}, \mathrm{H}_{2}\right), 7.41\left(\mathrm{t},{ }^{3} J=6 \mathrm{~Hz}, 2 \mathrm{H}, \mathrm{H}_{3^{3}}\right)$ $7.59\left(\mathrm{t},{ }^{3} J=6 \mathrm{~Hz}, 1 \mathrm{H}, \mathrm{H}_{5^{\prime \prime} / 6^{\prime \prime}}\right), 7.74\left(\mathrm{t},{ }^{3} J=6 \mathrm{~Hz}, 1 \mathrm{H}, \mathrm{H}_{5^{\prime \prime} / 6^{\prime}}\right), 7.90\left(\mathrm{~d},{ }^{3} J=6 \mathrm{~Hz}, 1 \mathrm{H}\right.$, $\left.\mathrm{H}_{6}\right), 8.00\left(\mathrm{~d},{ }^{3} J=6 \mathrm{~Hz}, 1 \mathrm{H}, \mathrm{H}_{7}\right), 8.14\left(\mathrm{~d},{ }^{3} J=6 \mathrm{~Hz}, 1 \mathrm{H}, \mathrm{H}_{5}\right), 8.55\left(\mathrm{~s}, 1 \mathrm{H}, \mathrm{H}_{4}\right) ;{ }^{13} \mathrm{C}$ 
$\operatorname{NMR}\left(75 \mathrm{MHz}, \mathrm{CDCl}_{3}\right) \delta: 106.2\left(\mathrm{C}_{3}\right), 111.7(\mathrm{C} \equiv \mathrm{N}), 115.1\left(\mathrm{C}_{8}\right), 116.3\left(\mathrm{C}_{10}\right), 121.0$ $\left(\mathrm{C}_{5^{\prime}}\right), 123.1\left(\mathrm{C}_{2^{\prime}}\right), 124.9\left(\mathrm{C}_{6^{\prime \prime}}\right), 126.4\left(\mathrm{C}_{5^{\prime \prime}}\right), 128.8\left(\mathrm{C}_{4^{\prime}}\right), 128.8\left(\mathrm{C}_{3^{\prime}}\right), 129.2\left(\mathrm{C}_{4}\right), 129.3$ $\left(\mathrm{C}_{7}\right), 130.3\left(\mathrm{C}_{6}\right), 135.3\left(\mathrm{C}_{6}\right), 140.4\left(\mathrm{C}_{5}\right), 144.6\left(\mathrm{C}_{1^{\prime}}\right), 144.8\left(\mathrm{C}_{2}\right), 153.8\left(\mathrm{C}_{9}\right)$. HRMS, $\mathrm{m} / z .319 .0840$ found (calculated for $\mathrm{C}_{26} \mathrm{H}_{12} \mathrm{~N}_{2} \mathrm{ONa}[\mathrm{M}+\mathrm{Na}]^{+}$requires 319.0842). Anal. Calcd for $\mathrm{C}_{20} \mathrm{H}_{12} \mathrm{~N}_{2} \mathrm{O}$ : C, 81.07; H, 4.08; N, 9.45. Found C, 81.08; H, 4.08; $\mathrm{N}, 9.44$.

2-(4-Methoxyphenylimino) -3 H-naphtho[2,1-b] pyran-2-carbonitrile (5d)

Yield $=80 \%$. Reddish powder, $\mathrm{mp}=232^{\circ} \mathrm{C}-234^{\circ} \mathrm{C} . \mathrm{IR}\left(\mathrm{cm}^{-1}\right): v=1650$ $(\mathrm{C}=\mathrm{N}), 2229(\mathrm{C} \equiv \mathrm{N}) ;{ }^{1} \mathrm{H}$ NMR $\left(300 \mathrm{MHz}, \mathrm{CDCl}_{3}\right) \delta: 3.87\left(\mathrm{~s}, 3 \mathrm{H}, \mathrm{H}_{4}{ }^{\prime \prime}\right), 6.95\left(\mathrm{~d},{ }^{3} J=\right.$ $\left.9 \mathrm{~Hz}, 2 \mathrm{H}, \mathrm{H}_{3^{\prime}}\right), 7.31\left(\mathrm{~d},{ }^{3} J=6 \mathrm{~Hz}, 1 \mathrm{H}, \mathrm{H}_{8}\right), 7.44\left(\mathrm{~d},{ }^{3} J=9 \mathrm{~Hz}, 2 \mathrm{H}, \mathrm{H}_{2^{\prime}}\right), 7.58\left(\mathrm{t},{ }^{3} J=\right.$ $\left.6 \mathrm{~Hz}, 1 \mathrm{H}, \mathrm{H}_{5^{\prime \prime} / 6^{\prime \prime}}\right), 7.72\left(\mathrm{t},{ }^{3} \mathrm{~J}=6 \mathrm{~Hz}, 1 \mathrm{H}, \mathrm{H}_{5^{\prime \prime} / 6^{\prime \prime}}\right), 7.90\left(\mathrm{~d},{ }^{3} \mathrm{~J}=6 \mathrm{~Hz}, 1 \mathrm{H}, \mathrm{H}_{6^{\prime}}\right), 8.00$ $\left(\mathrm{d},{ }^{3} \mathrm{~J}=6 \mathrm{~Hz}, 1 \mathrm{H}, \mathrm{H}_{7}\right), 8.12\left(\mathrm{~d},{ }^{3} \mathrm{~J}=6 \mathrm{~Hz}, 1 \mathrm{H}, \mathrm{H}_{5^{\prime}}\right), 8.47\left(\mathrm{~s}, 1 \mathrm{H}, \mathrm{H}_{4}\right) ;{ }^{13} \mathrm{C} \mathrm{NMR}(75$ $\left.\mathrm{MHz}, \mathrm{CDCl}_{3}\right) \delta: 55.5\left(\mathrm{C}_{4^{\prime}}\right), 106.8\left(\mathrm{C}_{3}\right), 111.7(\mathrm{C} \equiv \mathrm{N}), 113.9\left(\mathrm{C}_{3^{\prime}}\right), 115.2\left(\mathrm{C}_{8}\right), 116.3$ $\left(\mathrm{C}_{10}\right), 121.0\left(\mathrm{C}_{5^{\prime}}\right), 125.4\left(\mathrm{C}_{2^{\prime}}\right), 126.4\left(\mathrm{C}_{6}{ }^{\prime}\right), 128.8\left(\mathrm{C}_{5^{\prime}}\right), 129.1\left(\mathrm{C}_{4}\right), 129.1\left(\mathrm{C}_{7}\right), 130.3$ $\left(\mathrm{C}_{6}\right), 135.1\left(\mathrm{C}_{5}\right), 137.4\left(\mathrm{C}_{1^{\prime}}\right), 139.4\left(\mathrm{C}_{6}\right), 143.7\left(\mathrm{C}_{2}\right), 153.8\left(\mathrm{C}_{9}\right), 157.2\left(\mathrm{C}_{4^{\prime}}\right)$. HRMS, $\mathrm{m} / z .349 .0947$ found (calculated for $\mathrm{C}_{21} \mathrm{H}_{14} \mathrm{~N}_{2} \mathrm{O}_{2} \mathrm{Na}[\mathrm{M}+\mathrm{Na}]^{+}$requires 349.0948). Anal. Calcd for $\mathrm{C}_{21} \mathrm{H}_{14} \mathrm{~N}_{2} \mathrm{O}_{2}$ : C, 77.29; H, 4.32; N, 8.58. Found C, 77.31; H, 4.33; $\mathrm{N}, 8.57$.

Standard procedure for the synthesis of 2-N-phenylamino-4H-chromene3 -carbonitrile 6(a-d) by reduction of 2-N-phenylimino-2H-[1]-benzopyran-3carbonitrile $5(a-d)$.

In a $50 \mathrm{ml}$ round-bottomed flask, provided with a magnetic stirrer and condenser, containing a suspension of 2-phenylimino- $2 H$-[1]-benzopyran-3-carbonitrile 5 (12 mmol.) in $12 \mathrm{ml}$ of dry THF cooled at $0^{\circ} \mathrm{C}$ was added small portions of commercial sodium borohydride $(227 \mathrm{mg}, 6 \mathrm{mmol}$.). The resulting reaction mixture was stirred vigorously $(500 \mathrm{rpm})$ at $0{ }^{\circ} \mathrm{C}$ during 45 minutes. After stirring, the mixture was poured in $10 \mathrm{ml}$ of deionized water at room temperature without stirring to improve decantation and precipitation. The resulting precipitate was collected by filtration on a Büchner funnel (porosity $\mathrm{N}^{\circ} 4$ ) and washed with deionized water $(3 \times 10 \mathrm{ml})$. Recrystallization of the desired 2-phenylamino$4 H$-[1]-chromene-3-carbonitrile 6 was realized from a mixture of deionized water/ethanol, then dried under high vacuum $\left(10^{-2}\right.$ Torr $)$ at $25^{\circ} \mathrm{C}$ for 1 hour and afforded 6 as a powder.

\section{8-Methoxy-2-N-phenylamino-4H-chromene-3-carbonitrile (6a)}

Yield $=81 \%$. Yellowish powder, $\mathrm{mp}=122^{\circ} \mathrm{C}-124^{\circ} \mathrm{C}$; $\mathrm{IR}\left(\mathrm{cm}^{-1}\right): v=2195$ $(\mathrm{C} \equiv \mathrm{N}), 3320(\mathrm{NH}) ;{ }^{1} \mathrm{H} \mathrm{NMR}\left(300 \mathrm{MHz} \mathrm{CDCl}_{3}\right) \delta: 3.64\left(\mathrm{~s}, 2 \mathrm{H}, \mathrm{H}_{4}\right), 3.89(\mathrm{~s}, 3 \mathrm{H}$, $\left.\mathrm{OCH}_{3}\right), 6.61$ (br s, $\left.1 \mathrm{H}, \mathrm{NH}\right), 6.78\left(\mathrm{dd},{ }^{3} J=6 \mathrm{~Hz}, 2 \mathrm{H}, \mathrm{H}_{3^{\prime}}\right), 7.08\left(\mathrm{~m}, 2 \mathrm{H}, \mathrm{H}_{4^{\prime}}, \mathrm{H}_{7}\right)$, $7.34\left(\mathrm{~m}, 4 \mathrm{H}, \mathrm{H}_{5}, \mathrm{H}_{6}, \mathrm{H}_{2}\right) .{ }^{13} \mathrm{C} \mathrm{NMR}\left(75 \mathrm{MHz}, \mathrm{CDCl}_{3}\right) \delta: 24.6\left(\mathrm{C}_{4}\right), 56.2\left(\mathrm{OCH}_{3}\right)$, $56.8\left(\mathrm{C}_{3}\right), 110.7\left(\mathrm{C}_{7}\right), 119.8(\mathrm{C} \equiv \mathrm{N}), 119.9\left(\mathrm{C}_{2}\right), 120.1\left(\mathrm{C}_{4}\right), 120.6\left(\mathrm{C}_{5}\right), 123.4\left(\mathrm{C}_{10}\right)$, $125.0\left(\mathrm{C}_{6}\right), 129.1\left(\mathrm{C}_{3^{\prime}}\right), 138.1\left(\mathrm{C}_{1^{\prime}}\right), 139.1\left(\mathrm{C}_{9}\right), 148.1\left(\mathrm{C}_{2}\right), 157.8\left(\mathrm{C}_{8}\right)$. HRMS, $m / z$. 301.0948 found (calculated for $\mathrm{C}_{17} \mathrm{H}_{14} \mathrm{~N}_{2} \mathrm{O}_{2} \mathrm{Na}[\mathrm{M}+\mathrm{Na}]^{+}$requires 301.0947). Anal. Calcd for $\mathrm{C}_{17} \mathrm{H}_{14} \mathrm{~N}_{2} \mathrm{O}_{2}$ : C, 73.37; H, 5.07; N, 10.07. Found C, 73.35; H, 5.10; $\mathrm{N}, 10.09$.

8-Methoxy-2 -(4-methoxyphenylamino) -4H-chromene-3-carbonitrile (6b) 
Yield $=89 \%$. Yellowish powder, $\mathrm{mp}=148^{\circ} \mathrm{C}-150^{\circ} \mathrm{C}$; IR $\left(\mathrm{cm}^{-1}\right): v=2191$ (CN), $3232(\mathrm{NH}) ;{ }^{1} \mathrm{H}$ NMR (300 MHz, DMSO $\left.-d_{6}\right) \delta: 3.54\left(\mathrm{~s}, 2 \mathrm{H}, \mathrm{H}_{4}\right), 3.77(\mathrm{~s}, 3 \mathrm{H}$, $\left.\mathrm{H}_{4^{\prime \prime}}\right), 3.79\left(\mathrm{~s}, 3 \mathrm{H}, \mathrm{OCH}_{3}\right), 6.78\left(\mathrm{~d},{ }^{3} J=6 \mathrm{~Hz}, 1 \mathrm{H}, \mathrm{H}_{7}\right), 6.86\left(\mathrm{~d},{ }^{3} J=9 \mathrm{~Hz}, 2 \mathrm{H}, \mathrm{H}_{2^{\prime}}\right)$, $6.97\left(\mathrm{~d},{ }^{3} J=6 \mathrm{~Hz}, 1 \mathrm{H}, \mathrm{H}_{5}\right), 7.09\left(\mathrm{t},{ }^{3} J=6 \mathrm{~Hz}, 1 \mathrm{H}, \mathrm{H}_{6}\right), 7.15\left(\mathrm{~d},{ }^{3} J=9 \mathrm{~Hz}, 1 \mathrm{H}, \mathrm{H}_{3^{\prime}}\right)$, 9.16 (br s, $1 \mathrm{H}, \mathrm{NH}) .{ }^{13} \mathrm{C}$ NMR $\left(75 \mathrm{MHz}, \mathrm{DMSO}-d_{6}\right) \delta: 25.3\left(\mathrm{C}_{4}\right), 55.7\left(\mathrm{C}_{4^{\prime}}\right), 56.5$ $\left(\mathrm{OCH}_{3}\right), 56.9\left(\mathrm{C}_{3}\right), 111.6\left(\mathrm{C}_{7}\right), 114.4\left(\mathrm{C}_{3^{3}}\right), 120.1(\mathrm{C} \equiv \mathrm{N}), 120.3\left(\mathrm{C}_{5}\right), 121.9\left(\mathrm{C}_{10}\right)$, $122.2\left(\mathrm{C}_{2^{\prime}}\right), 125.3\left(\mathrm{C}_{6}\right), 132.6\left(\mathrm{C}_{1^{\prime}}\right), 139.2\left(\mathrm{C}_{9}\right), 148.1\left(\mathrm{C}_{2}\right), 155.5\left(\mathrm{C}_{8}\right), 157.9\left(\mathrm{C}_{4^{\prime}}\right)$. HRMS, $m / z .331 .1056$ found (calculated for $\mathrm{C}_{18} \mathrm{H}_{16} \mathrm{~N}_{2} \mathrm{O}_{3} \mathrm{Na}[\mathrm{M}+\mathrm{Na}]^{+}$requires 331.1053). Anal. Calcd for $\mathrm{C}_{18} \mathrm{H}_{16} \mathrm{~N}_{2} \mathrm{O}_{3}$ : C, 70.12; H, 5.23; N, 9.09. Found $\mathrm{C}$, 70.14; H, 5.22; N, 9.11.

\section{2-N-Phenylamino-4H-benzo[ $h]$ chromene-3-carbonitrile (6c)}

Yield $=84 \%$. Yellowish powder, $\mathrm{mp}=188^{\circ} \mathrm{C}-190^{\circ} \mathrm{C}$. IR $\left(\mathrm{cm}^{-1}\right): v=2193$ $(\mathrm{C} \equiv \mathrm{N}), 3278(\mathrm{NH}) ;{ }^{1} \mathrm{H} \mathrm{NMR}\left(300 \mathrm{MHz}, \mathrm{CDCl}_{3}\right) \delta: 3.98\left(\mathrm{~s}, 2 \mathrm{H}, \mathrm{H}_{4}\right), 6.66$ (br s, $1 \mathrm{H}, \mathrm{NH}), 7.17\left(\mathrm{~m}, 2 \mathrm{H}, \mathrm{H}_{8}, \mathrm{H}_{4^{\prime}}\right), 7.23\left(\mathrm{~d},{ }^{3} J=6 \mathrm{~Hz}, 2 \mathrm{H}, \mathrm{H}_{2^{\prime}}\right), 7.40\left(\mathrm{t},{ }^{3} J=6 \mathrm{~Hz}, 2 \mathrm{H}\right.$, $\left.\mathrm{H}_{3^{3}}\right), 7.53\left(\mathrm{t},{ }^{3} \mathrm{~J}=6 \mathrm{~Hz}, 1 \mathrm{H}, \mathrm{H}_{5^{\prime \prime} / 6^{\prime \prime}}\right), 7.64\left(\mathrm{t},{ }^{3} J=6 \mathrm{~Hz}, 1 \mathrm{H}, \mathrm{H}_{5^{\prime \prime} / 6^{\prime \prime}}\right), 7.78\left(\mathrm{t},{ }^{3} J=6 \mathrm{~Hz}\right.$, $\left.2 \mathrm{H}, \mathrm{H}_{7}, \mathrm{H}_{6}\right), 7.87\left(\mathrm{~d},{ }^{3} J=6 \mathrm{~Hz}, 1 \mathrm{H}, \mathrm{H}_{5}\right) .{ }^{13} \mathrm{C} \mathrm{NMR}\left(75 \mathrm{MHz}, \mathrm{CDCl}_{3}\right) \delta: 22.5\left(\mathrm{C}_{4}\right)$, $57.66\left(\mathrm{C}_{3}\right), 111.7\left(\mathrm{C}_{8}\right), 116.8\left(\mathrm{C}_{10}\right), 120.3(\mathrm{C} \equiv \mathrm{N}), 121.0\left(\mathrm{C}_{2}\right), 122.7\left(\mathrm{C}_{7}\right), 124.1$ $\left(\mathrm{C}_{5^{\prime}}\right), 125.4\left(\mathrm{C}_{4^{\prime}}\right), 127.4\left(\mathrm{C}_{6^{\prime}}\right), 128.5\left(\mathrm{C}_{5^{\prime \prime}}\right), 128.9\left(\mathrm{C}_{6}\right), 130.9\left(\mathrm{C}_{5}\right), 131.1\left(\mathrm{C}_{6}\right), 137.7$ $\left(\mathrm{C}_{1}\right), 146.4\left(\mathrm{C}_{2}\right), 157.2\left(\mathrm{C}_{9}\right)$. HRMS, $\mathrm{m} / \mathrm{z} .321 .0998$ found (calculated for $\mathrm{C}_{20} \mathrm{H}_{14} \mathrm{~N}_{2} \mathrm{ONa}[\mathrm{M}+\mathrm{Na}]^{+}$requires 321.09983). Anal. Calcd for $\mathrm{C}_{20} \mathrm{H}_{14} \mathrm{~N}_{2} \mathrm{O}$ : C, 80.52; H, 4.73; N, 9.39. Found C, 80.56; H, 4.71; N, 9.38.

\section{2-(4-Methoxyphenylamino) -4H-benzo[h] chromene-3-carbonitrile (6d)}

Yield $=85 \%$. Yellowish powder, $\mathrm{mp}=200^{\circ} \mathrm{C}-202^{\circ} \mathrm{C}$; IR $\left(\mathrm{cm}^{-1}\right): v=2189$ $(\mathrm{C} \equiv \mathrm{N}), 3235(\mathrm{NH}) ;{ }^{1} \mathrm{H}$ NMR $\left(300 \mathrm{MHz}, \mathrm{CDCl}_{3}\right) \delta: 3.86\left(\mathrm{~s}, 3 \mathrm{H}, \mathrm{H}_{4^{\prime}}\right), 3.96(\mathrm{~s}, 2 \mathrm{H}$, $\left.\mathrm{C}_{4}\right), 6.51(\mathrm{br} \mathrm{s}, 1 \mathrm{H}, \mathrm{NH}), 6.94\left(\mathrm{~d},{ }^{3} J=6 \mathrm{~Hz}, 2 \mathrm{H}, \mathrm{H}_{2}\right), 7.11\left(\mathrm{~d},{ }^{3} J=6 \mathrm{~Hz}, 2 \mathrm{H}, \mathrm{H}_{8}\right.$ ), $7.18\left(\mathrm{t},{ }^{3} J=6 \mathrm{~Hz}, 2 \mathrm{H}, \mathrm{H}_{3^{3}}\right), 7.52\left(\mathrm{t},{ }^{3} J=6 \mathrm{~Hz}, 1 \mathrm{H}, \mathrm{H}_{5^{\prime \prime} / 6^{\prime \prime}}\right), 7.63\left(\mathrm{t},{ }^{3} J=6 \mathrm{~Hz}, 1 \mathrm{H}\right.$, $\left.\mathrm{H}_{5^{\prime \prime} / 6^{\prime}}\right), 7.77\left(\mathrm{dd},{ }^{3} \mathrm{~J}=6 \mathrm{~Hz}, 2 \mathrm{H}, \mathrm{H}_{7}, \mathrm{H}_{6}\right), 7.86\left(\mathrm{~d},{ }^{3} \mathrm{~J}=6 \mathrm{~Hz}, 1 \mathrm{H}, \mathrm{H}_{5}\right) ;{ }^{13} \mathrm{C} \mathrm{NMR}(75$ $\left.\mathrm{MHz}, \mathrm{CDCl}_{3}\right) \delta: 22.4\left(\mathrm{C}_{4}\right), 55.5\left(\mathrm{C}_{4^{\prime \prime}}\right), 111.9\left(\mathrm{C}_{3}\right), 114.4\left(\mathrm{C}_{3}\right), 116.8\left(\mathrm{C}_{8}\right), 120.6$ $\left(\mathrm{C}_{10}\right), 122.7\left(\mathrm{C}_{\mathrm{N}}\right), 124.1\left(\mathrm{C}_{2}\right), 125.3\left(\mathrm{C}_{7}\right), 127.3\left(\mathrm{C}_{5^{\prime}}\right), 128.5\left(\mathrm{C}_{6^{\prime}}\right), 128.8\left(\mathrm{C}_{5^{\prime \prime}}\right), 130.3$ $\left(\mathrm{C}_{6}\right), 130.9\left(\mathrm{C}_{5}\right), 131.2\left(\mathrm{C}_{6}\right), 146.4\left(\mathrm{C}_{2}\right), 156.9\left(\mathrm{C}_{9}\right), 157.8\left(\mathrm{C}_{4}\right)$. HRMS, m/z. 351.1105 found (calculated for $\mathrm{C}_{21} \mathrm{H}_{16} \mathrm{~N}_{2} \mathrm{O}_{2} \mathrm{Na}[\mathrm{M}+\mathrm{Na}]^{+}$requires 351.1104). Anal. Calcd for $\mathrm{C}_{21} \mathrm{H}_{16} \mathrm{~N}_{2} \mathrm{O}_{2}$ : C, 76.81; H, 4.91; N, 8.53. Found C, 76.79; H, 4.90; N, 8.52 .

\subsection{Biochemistry}

\subsubsection{Protein Kinase Preparations and Assays}

Kinase activities were assayed in:

- in buffer (A): $10 \mathrm{mM} \mathrm{MgCl}$, 1 mM EGTA, $1 \mathrm{mM}$ DTT, $25 \mathrm{mM}$ Tris-HCl pH $7.5,50 \mu \mathrm{g} / \mathrm{ml}$ heparin

- or in buffer (B): $60 \mathrm{mM} \beta$-glycerophosphate, $30 \mathrm{mM}$ p-nitrophenyl-phosphate, $25 \mathrm{mM}$ MOPS (pH 7), 5 mM EGTA, $15 \mathrm{mM} \mathrm{MgCl}$, $1 \mathrm{mM}$ DTT, $0.1 \mathrm{mM}$ sodium orthovanadate

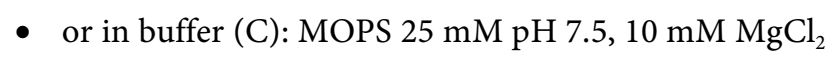

with their corresponding substrate in the presence of $15 \mu \mathrm{M}\left[\gamma^{-33} \mathrm{P}\right]$ ATP (3000 
$\mathrm{Ci} / \mathrm{mmol} ; 10 \mathrm{mCi} / \mathrm{ml}$ ) in a final volume of $30 \mu \mathrm{l}$ following the assay described in [31]. Controls were performed with appropriate dilutions of dimethylsulfoxid. Full-length kinases are used unless specified. Peptide substrates were obtained from Proteogenix (Oberhausbergen, France)

- $H_{s} \mathrm{CDK} 5 / \mathrm{p} 25$ (human, recombinant, expressed in bacteria) was assayed in buffer B, with $0.8 \mu \mathrm{g} / \mu \mathrm{l}$ of histone $\mathrm{H} 1$ as substrate;

- $H_{s} \mathrm{CDK} 5 / \mathrm{p} 25$ (human, recombinant, expressed in bacteria) was assayed in buffer B, with $0.8 \mu \mathrm{g} / \mu \mathrm{l}$ of histone $\mathrm{H} 1$ as substrate;

- $\underline{S s C K 1 \mathcal{E}}$ (casein kinase $1 \mathcal{E}$, porcine brain, native, affinity purified) was assayed in buffer B, with $0.022 \mu \mathrm{g} / \mu \mathrm{l}$ of the following peptide: RRKHAAIGSpAYSITA as CK1-specific substrate;

- $S s$ GGSK-3 $\beta$ (glycogen synthase kinase-3, porcine brain, native, affinity purified) was assayed in buffer A $(+0.15 \mathrm{mg} / \mathrm{ml}$ of BSA $+0.23 \mathrm{mg} / \mathrm{ml}$ of DTT), with $0.010 \mu \mathrm{g} / \mu \mathrm{l}$ of GS-1 peptide, a GSK-3-selective substrate (YRRAAVPPSPSLSRHSSPHQSpEDEEE, "Sp" stands for phosphorylated serine);

- HsHaspin-kd (human, kinase domain, amino acids 470 to 798, recombinant, expressed in bacteria) was assayed in buffer $\mathrm{C}$ with $0.007 \mu \mathrm{g} / \mu \mathrm{l}$ of Histone $\mathrm{H} 3$ (1-21) peptide (ARTKQTARKSTGGKAPRKQLA) as substrate;

- HsPIM1 (human proto-oncogene, recombinant, expressed in bacteria) was assayed in buffer B with $0.8 \mu \mathrm{g} / \mu \mathrm{l}$ of histone H1 (Sigma \#H5505) as substrate;

- MmCLK1 (from Mus musculus, recombinant, expressed in bacteria) was assayed in buffer A $(+0.15 \mathrm{mg} / \mathrm{ml}$ of BSA $+0.23 \mathrm{mg} / \mathrm{ml}$ of DTT) with 0.027 $\mu \mathrm{g} / \mu \mathrm{l}$ of the following peptide: GRSRSRSRSRSR as substrate;

- $R n$ DYRK1A-kd (Rattus norvegicus, amino acids 1 to 499 including the kinase domain, recombinant, expressed in bacteria, DNA vector kindly provided by Dr. W. Becker, Aachen, Germany) was assayed in buffer A $(+0.5$ $\mathrm{mg} / \mathrm{ml}$ of BSA $+0.23 \mathrm{mg} / \mathrm{ml}$ of DTT) with $0.033 \mu \mathrm{g} / \mu \mathrm{l}$ of the following peptide: KKISGRLSPIMTEQ as substrate.

\subsubsection{Cell Culture and Survival Assays}

Huh-7D12 (differential hepatocellular carcinoma, Ref ECACC: 01042712), Caco2 (differentiated colorectal adenocarcinoma, Ref ECACC: 86010202), MDA-MB-231 (breast carcinoma, Ref ECACC: 92020424), HCT-116 (actively proliferating colorectal adenocarcinoma, Ref ECACC: 91091005), PC3 (prostate carcinoma, Ref ECACC: 90112714), NCI-H727 (lung carcinoma, Ref ECACC: 94060303) cell lines were obtained from the ECACC collection and $\mathrm{HaCaT}$ (keratinocyte from Cell Lines Service, Eppelheim, Germany). Cells were grown according to ECACC recommendations [32]. The toxicity test of the compounds on these cells was as follows: $2 \times 10^{3}$ cells for HCT-116 cells or $4 \times 10^{3}$ for the other cells were seeded in 96 multi well plates in triplicate and left for $24 \mathrm{~h}$ for attachment, spreading and growing. Then, cells were exposed for $48 \mathrm{~h}$ to increasing concentrations of the compounds, ranging from 0.1 to $25 \mathrm{mM}$ in a final volume of $120 \mathrm{ml}$ of culture medium. Cells were fixed in cooled ethanol-acetic acid solution (95:5), nuclei were stained with Hoechst 3342 (Sigma) and counted using automated imag- 
ing analysis (Cellomics Arrayscan VTI/HCS Reader, Thermo/Scientific).

\section{Acknowledgements}

One of us (A.B.) wishes to thank the "Ministère de l'Enseignement Supérieur et de la Recherche de Tunisie" for the grant. Financial support of this program carried out under the "Cancéropôle Grand Ouest" in "Molécules Marines, Métabolisme et Cancer" network, is gratefully acknowledged. The authors are grateful to the assistance of the staff of the CRMPO analytical chemistry core facility for HRMS and centesimal analysis (CRMPO platform ScanMAT UMS 2001 CNRS, Université de Rennes 1, Bat. 11A, Campus de Beaulieu, Rennes, France).

\section{Conflicts of Interest}

The authors declare no conflict of interest.

\section{References}

[1] Patil, S.A., Patil, R., Pfeffer, L.M. and Miller, D.D. (2013) Chromenes: Potential New Chemotherapeutic Agents for Cancer. Future Medicinal Chemistry, 14, 1647-1660. https://doi.org/10.4155/fmc.13.126

[2] Cai, S.X., Drewe, J.A., Kasibhatla, S., Kemnitzer, W.E., Tseng, B.Y., Blais, C., Gourdeau, H. and Labrecque, D. (2008) Substituted 4-Aryl-chromene as Caspase Activator and Apoptosis Inducer, and as Antivascular Agent for Treatment of Diseases Due to Vasculature Overgrowth, Such as Solid Tumors and Ocular Neovascularization. Patent WO2008/005572 A2, 6 July 2006.

[3] Kemnitzer, W., Drewe, J., Jiang, S., Zhang, H., Zhao, J., Crogan-Grundy, C., Xu, L., Lamothe, S., Gourdeau, H., Denis, R., Ben, T., Shailaja, K. and Cai, S.X. (2007) Discovery of 4-Aryl-4H-chromenes as a New Series of Apoptosis Inducers Using a Cell- and Caspase-Based High-Throughput Screening Assay. 3. Structure-Activity Relationships of Fused Rings at the 7,8-Positions. Journal of Medicinal Chemistry, 50, 2858-2864. https://doi.org/10.1021/jm070216c

[4] Kemnitzer, W., Shailaja, K., Songchun, J., Zhang, Z., Zhao, J., Jia, S., Xu, L., Crogan-Grundy, C., Denis, R., Barriault, N., Vaillancourt, L., Charron, S., Dodd, J., Attardo, G., Labrecque, D., Lamothe, S., Gourdeau, H., Tseng, B., Drewe, J. and Cai, S.X. (2005) Discovery of 4-Aryl-4H-chromenes as a New Series of Apoptosis Inducers Using a Cell- and Caspase-Based High-Throughput Screening Assay. 2. Structure-Activity Relationships of the 7- and 5-, 6-, 8-Positions. Bioorganic \& $\mathrm{Me}$ dicinal Chemistry Letters, 15, 4745-4751. https://doi.org/10.1016/j.bmcl.2005.07.066

[5] Gulley J.M.D. (2010) A Phase I/II Trial of Crolibulin (EPC2407) plus Cisplatin in Adults with Solid Tumors with a Focus on Anaplastic Thyroid Cancer (ATC). http://www.clinicaltrials.gov

[6] Kasibhatla, S., Gourdeau, H., Meerovitch, K., Drewe, J., Reddy, S., Qiu, L., Zhang, H., Bergeron, F., Bouffard, D., Yang, Q., Herich, J., Lamothe, S., Cai, S.X. and Tseng, B. (2004) Discovery and Mechanism of Action of a Novel Series of Apoptosis Inducers with Potential Vascular Targeting Activity. Molecular Cancer Therapeutics, 11, 1365-1374

[7] Gourdeau, H., Leblond, L., Hamelin, B., Desputeau, C., Dong, K., Kianicka, I., Custeau, D., Boudreau, C., Geerts, L., Cai, S.X., Drewe, J., Labrecque, D., Kasibhatla, S. and Tseng, B. (2004) Antivascular and Antitumor Evaluation of 2-Amino-4-(3- 
bromo-4,5-dimethoxy-phenyl)-3-cyano-4H-chromenes, a Novel Series of Anticancer Agents. Molecular Cancer Therapeutics, 11, 1375-1384.

[8] Manero, F., Gautier, F., Gallenne, T., Cauquil, N., Grée, D., Cartron, P.-F., Geneste, O., Rene Grée, R., Vallette, F.M. and Juin, P. (2006) The Small Organic Compound HA14-1 Prevents Bcl-2 Interaction with Bax to Sensitize Malignant Glioma Cells to Induction of Cell Death. Cancer Research, 66, 2757-2764.

https://doi.org/10.1158/0008-5472.CAN-05-2097

[9] Doshi, J.M., Tian, D. and Xing, C. (2006) Structure Activity Relationship Studies of Ethyl-2-amino-6-bromo-4-(1-cyano-2-ethoxy-2-oxoethyl)-4H-chromene-3-carboxy late (HA 14-1), an Antagonist for Antiapoptotic Bcl-2 Proteins to Overcome Drug Resistance in Cancer. Journal of Medicinal Chemistry, 49, 7731-7739.

https://doi.org/10.1021/jm060968r

[10] Grée, D., Vorin, S., Manthati, V.L., Caijo, F., Guillaume Viault, G., Manero, F., Juin, P. and Grée, R. (2008) The Synthesis of New, Selected Analogues of the Pro-Apoptotic and Anticancer Molecule HA 14-1. Tetrahedron Letters, 49, 3276-3278. https://doi.org/10.1016/j.tetlet.2008.03.070

[11] Wang, J.-L., Liu, D., Zhang, Z.-J., Shan, S., Han, X., Srinivasula, S.M., Croce, C.M., Alnemri, E.S. and Huang, Z. (2000) Structure-Based Discovery of an Organic Compound That Binds Bcl-2 Protein and Induces Apoptosis of Tumor Cells. PNAS, 97, 7124-7129. https://doi.org/10.1073/pnas.97.13.7124

[12] Weyland, M., Manero, F., Paillard, A., Grée, D., Viault, G., Jarnet, D., Menei, P., Juin, P., Chourpa, I., Benoit, J.-P., Grée, R. and Garcion, E. (2011) Mitochondrial Targeting by Use of Lipid Nanocapsules Loaded with SV30, an Analogue of the Small-Molecule Bcl-2 Inhibitor HA 14-1. Journal of Controlled Release, 151, 74-82. https://doi.org/10.1016/j.jconrel.2010.11.032

[13] Wood, D.L., Panda, D., Wiernicki, T.R., Wilson, L., Jordan, M.A. and Singh, J.P. (1997) Inhibition of Mitosis and Microtubule Function through Direct Tubulin Binding by a Novel Antiproliferative Naphthopyran LY290181. Molecular Pharmaceutics, 52, 437-444. https://doi.org/10.1124/mol.52.3.437

[14] Panda, D., Singh, J.P. and Wilson, L. (1997) Suppression of Microtubule Dynamics by LY290181. The Journal of Biological Chemistry, 272, 7681-7687. https://doi.org/10.1074/jbc.272.12.7681

[15] Sciabola, S., Carosati, E., Cucurull-Sanchez, L., Baronic, M. and Mannhold, R. (2007) Novel TOPP Descriptors in 3D-QSAR Analysis of Apoptosis Inducing 4-Aryl-4H-chromenes: Comparison versus Other 2D- and 3D-Descriptors. Bioorganic \& Medicinal Chemistry, 15, 6450-6462. https://doi.org/10.1016/j.bmc.2007.06.051

[16] Souchet, B., Audrain, M., Billard, J-M; Dairou, J., Fol, R., Orefice, N., Tada, S., Dufayet, G., Alves, S., Potier, B., Dutar, P., Limanton, E., Carreaux, F., Bazureau, J.-P., Meijer, L., Janelle, N., Braudeau, J. and Cartier, N. (2019) Inhibition of DYRK1A Proteolysis Modifies Its Kinase Specificity and Rescues Alzheimer Phenotype in APP/PS1 Mice. Acta Neuropathologica Communications, 7, Article 46. https://doi.org/10.1186/s40478-019-0678-6

[17] Nguyen, T.L., Duchon, A., Manousopoulou, A., Loaëc, N., Villiers, B., Pani, G., Harsan, L., Limanton, E., Bazureau, J.-P., Carreaux, F., Garbis, S.D., Meijer, L. and Herault, Y. (2018) Correction of Cognitive Deficits in Mouse Models of Down Syndrome by Pharmacological Inhibitor of DYRK1A. Disease Models \& Mechanisms, 11, dmm035634. https://doi.org/10.1242/dmm.035634

[18] Dago, C.D., Le Maux, P., Roisnel, T., Brigaudeau, C., Bekro, Y.-A., Mignen, O. and Bazureau, J.-P. (2018) Preliminary Structure-Activity Relationship (SAR) of a Novel 
Series of Pyrazole SKF-96365 Analogues as Potential Store-Operated Calcium Entry (SOCE) Inhibitors. International Journal of Molecular Sciences, 19, 856-879. https://doi.org/10.3390/ijms19030856

[19] Bouattour, A., Fakhfakh, M., Abid, S., Paquin, L., Le Guével, R., Corlu, A., Ruchaud, S., Bach, S., Ammar, H. and Bazureau, J.-P. (2017) Microwave Assisted a Practical Synthesis of 4-Imino-3-phenyl-3,4-dihydro-1 $H$-chromeno[2,3-d]pyrimidine-2(5H)thione Derivatives and Exploration of Their Biological Activities. Arkivoc, 4, 291-302. https://doi.org/10.24820/ark.5550190.p010.040

[20] Bouattour, A., Fakhfakh, M., Abid, S., Paquin, L., Ammar, H. and Bazureau, J.-P. (2017) An Efficient and Concise Syntheses of $N$-3-Substituted 9-Methoxy-4H-[1]benzopyrano[2,3-d]pyrimidine-4(5H)-imine and Formamidine Derivatives from Methyl $N$-(3-Cyano-8-methoxy-4H-[1]-benzopyran-2-yl)methanimidate. Synthesis, 49, 3768-3774. https://doi.org/10.1055/s-0036-1588825

[21] Fakhfakh, M., Turki, H., Fery-Forgues, S. and El Gharbi, R. (2010) The Synthesis and Optical Properties of Novel Fluorescent Iminocoumarins and Bis-Iminocoumarins: Investigations in the Series of Urea Derivatives. Dyes \& Pigments, 84, 108-113. https://doi.org/10.1016/j.dyepig.2009.07.003

[22] Ben Maktouf, L., Kammoun, M., Ammar, H. and Abid, S. (2011) Reactivité des 3-cyano iminocoumarines vis-à-vis du phénylsemicarbazide. Journal Society Chemistry Tunisia, 13, 101-106. http://www.sctunisie.org/journal-sct/index.php?categorie15/volume-13-dec-2011

[23] Benhaoua, C., Kasmi, S., Rahmouni, M. and Bazureau, J.-P. (2018) Synthesis of Some Enaminone Derivatives under Solvent-Free Process. Moroccan Journal of Heterocyclic Chemistry, 17, 50-60.

[24] Leclerc, S., Garnier, M., Hoessel, R., Marko, D., Bibb, J.A., Snyder, G.L., Greengard, P., Biernat, J., Mandelkow, E.M., Eisenbrand, G. and Meijer, L. (2001) Indirubins Inhibit Glycogen Synthase Kinase- $3 \beta$ and CDK5/P25, Two Protein Kinases Involved in Abnormal Tau Phosphorylation in Alzheimer's Disease. A Property Common to Most Cyclin Dependent Kinase Inhibitors? The Journal of Biological Chemistry, 276, 251-260. https://doi.org/10.1074/jbc.M002466200

[25] Sonawane, Y.A., Taylor, M.A., Napoleon, J.V., Rana, S., Contreras, J.I. and Natarajan, A. (2016) Cyclin Dependent Kinase 9 Inhibitors for Cancer Therapy. Journal of Medicinal Chemistry, 59, 8667-8684.

https://doi.org/10.1021/acs.jmedchem.6b00150

[26] Rena, G., Bain, J., Elliott, M. and Cohen, P. (2004) D4476, a Cell-Permeant Inhibitor of CK1, Suppresses the Site-Specific Phosphorylation and Nuclear Exclusion of FOXO1a. EMBO Reports, 5, 60-65. https://doi.org/10.1038/sj.embor.7400048

[27] Primot, A., Baratte, B., Gompel, M., Borgne, A., Liabeuf, S., Romette, J.L., Costantini, F. and Meijer, L. (2000) Purification of GSK-3 by Affinity Chromatography on Immobilized Axin. Protein Expression and Purification, 20, 394-404. https://doi.org/10.1006/prep.2000.1321

[28] Blanco-Aparicio, C. and Carnero, A. (2013) Pim Kinases in Cancer: Diagnostic, Prognostic and Treatment Opportunities. Biochemical Pharmacology, 85, 629-643. https://doi.org/10.1016/j.bcp.2012.09.018

[29] Karthikeyan, J.P., Moorthy, N.S., Waiker, D.K., Jain, A.K. and TRivedi, P. (2014) Human CDC2-Like Kinase 1 (CLK1): A Novel Target for Alzheimer's Disease. Current Drug Targets, 15, 539-550. https://doi.org/10.2174/1389450115666140226112321

[30] Fant, X., Durieu, E., Chicanne, G., Payrastre, B., Sbrissa, D., Shisheva, A., Limanton, 
E., Carreaux, F., Bazureau, J.-P. and Meijer, L. (2014) CLK/DYRK Kinases Inhibitor Leucettine L41 Induces mTOR-Dependent Autophagy. Implication for Alzheimers' Disease. Molecular Pharmaceutics, 85, 441-450.

https://doi.org/10.1124/mol.113.090837

[31] Bach, S., Knockaert, M., Reinhardt, J., Lozach, O., Schmitt, S., Baratte, B., Koken, M., Coburn, S.P., Tang, L., Jiang, T., Liang, D.C., Galons, H., Dierick, J.-F., Pinna, L.A., Meggio, F., Totzke, F., Schachtele, C., Lerman, A.S., Carnero, A., Wan, Y., Gray, N. and Meijer, L. (2005) Roscovitine Targets, Protein Kinases and Pyridoxal Kinase. The Journal of Biological Chemistry, 280, 31208-31219.

https://doi.org/10.1074/jbc.M500806200

[32] Nakabayashi, H., Taketssa, K., Miyano, K., Yamane, T. and Sato, J. (1982) Growth of Human Hepatoma Cell Lines with Differentiated Functions in Chemically Defined Medium. Cancer Research, 42, 3858-3863. 